\title{
Short pulse multi-frequency phase-based time delay estimation
}

\author{
Said Assous, ${ }^{\text {a) }}$ Clare Hopper, and Mike Lovell \\ Department of Geology, Ultrasound Research Laboratory, University of Leicester, Leicester LE1 7RH, \\ United Kingdom \\ Dave Gunn, Peter Jackson, and John Rees \\ Ultrasound Research Laboratory British Geological Survey, Keyworth NG12 5GG, United Kingdom
}

(Received 6 January 2009; revised 9 October 2009; accepted 9 October 2009)

\begin{abstract}
An approach for time delay estimation, based on phase difference detection, is presented. A multiple-frequency short continuous wave pulse is used to solve the well-known phase ambiguity problem when the maximum distance exceeds a full wavelength. Within an unambiguous range defined with the lowest frequency difference between components, the corresponding phase difference is unique and any distance within this range can be determined. Phase differences between higher frequency components are used to achieve a finer resolution. The concept will be presented and the effectiveness of the approach will be investigated through theoretical and practical examples. The method will be validated using underwater acoustic measurements, simulating noisy environments, demonstrating resolutions better than a 50th of a wavelength, even in the presence of high levels $(-5 \mathrm{~dB})$ of additive Gaussian noise. Furthermore, the algorithm is simple to use and can be easily implemented, being based on phase detection using the discrete Fourier transform.
\end{abstract}

\section{INTRODUCTION}

Time delay estimation is a fundamental step in source localization or beamforming applications and has attracted considerable research attention over the past few decades in many fields including radar, sonar, seismology, geophysics, ultrasonics, communication, and medical imaging. Various techniques are reported in the literature $e^{1-5}$ and a state of the art review can be found in Ref. 6 which concerns critical techniques, limitations, and recent advances that have significantly improved the performance of time delay estimation in adverse environments. These techniques can be classified into two broad categories: correlator-based approaches and system-identification-based techniques. Both categories can be implemented using two or more sensors; in general, more sensors lead to increase robustness due to greater redundancy. When the time delay is not an integral multiple of the sampling rate, however, it is necessary to either increase the sampling rate or use interpolation. ${ }^{6}$ In this paper, we present a new approach for time delay estimation, based on the received signal phase information. This avoids barriers encountered by alternative approaches based on phase information, which are limited by the need to use a reference signal, usually provided by a coherent local oscillator. ${ }^{7}$ Ambiguities in such phase measurement, caused by the inability to count integer number of cycles (wavelengths), are resolved using the Chinese remainder theorem (CRT) taken from number theory, where wavelength selection is based on pair-wise relatively prime wavelengths. ${ }^{7,8}$ However, the CRT is not entirely robust, in the sense that small errors in its remainders may induce a large error in the determined integer. ${ }^{9,10}$

\footnotetext{
a) Author to whom correspondence should be addressed. Electronic mail: sa251@le.ac.uk.
}

Another phase-based measurement approach, adopted to ensure accurate positioning of commercial robots, uses two or more frequencies in a decade scale in a transmitted signal. In this, the phase shift of the received signal with respect to the transmitted signal is exploited for ranging. ${ }^{11,12}$ However, this approach is valid only when the maximum pathlength/ displacement is less than one wavelength, otherwise a phase ambiguity will appear.

The time delay estimation approach proposed here is based on the use of local phase differences between specific frequency components of a short continuous wave (cw) received signal pulse. Within an unambiguous range defined by the lowest frequency difference between components, the corresponding phase difference is unique and any distance within this range can be determined. Phase differences between higher frequency components are used to achieve a finer resolution. Thus our approach overcomes the need to cross-correlate the received signal with either a reference signal or the transmitted signal. Unlike conventional methods, this approach is not limited by phase ambiguity; therefore most practical situations, where the range to be determined is beyond one wavelength, can be accommodated.

\section{METHODOLOGY}

\section{A. Concept}

The inspiration for development of the technique comes from the observation that bats have been shown to have exceptional resolution with regard to target detection when searching during flight. ${ }^{13-15} \mathrm{Au}$ and Simmons, ${ }^{15}$ somewhat controversially, concluded that bats using pulses with a center frequency of about $80 \mathrm{kHz}$ (40 kHz bandwidth) can achieve a distance resolution in air approaching $20 \mu \mathrm{m}$. At 
this frequency, and using $\lambda / 2$ as the guide for resolution, we see that the resolution reported by Au and Simmons is about 200 times better than that predicted conventionally. We sought to develop a methodology which would allow us to approach a resolution comparable to that achieved by such creatures which is still better than any man-made system.

Consider an acoustic pulse containing a single frequency component $f_{1}$ with an initial zero phase offset. This pulse is emitted through the medium, impinges on a target, is reflected and returns. The signal is captured and its phase measured relative to the transmitted pulse. Given this situation, we cannot estimate the distance to and from an object greater than one wavelength away (hence, usually, we would estimate the time of arrival of the pulse and assume a value for the velocity of sound in the medium to estimate the distance to the target).

For simplicity, assume the pulse contains a single cycle of frequency $f_{1}$ of wavelength $\lambda_{1}$. The distance $D$ to the target can be expressed as

$$
D=n_{1} \lambda_{1}+r_{1},
$$

where $\lambda_{1}=v / f_{1}, n_{1}$ is an integer, $r_{1}$ is a fraction of the wavelength $\lambda_{1}$, and $v$ is the speed of sound in the medium.

$r_{1}$ can be expressed as follows:

$$
r_{1}=\lambda_{1} \times \frac{\phi_{1}}{360},
$$

where $\phi_{1}$ is the residual phase angle in degrees. Combining Eqs. (1) and (2) and rearranging

$$
\begin{aligned}
& D=n_{1} \lambda_{1}+\lambda_{1} \frac{\phi_{1}}{360}, \\
& =n_{1} \frac{v}{f_{1}}+\frac{\phi_{1}}{360} \frac{v}{f_{1}}, \\
& D=\frac{v}{f_{1}}\left(n_{1}+\frac{\phi_{1}}{360}\right) .
\end{aligned}
$$

If we transmit a second frequency component $f_{2}$ within the same pulse, then it will also have associated with it a wavelength $\lambda_{2}$ and a residual phase $\phi_{2}$; similarly,

$$
D=\frac{v}{f_{2}}\left(n_{2}+\frac{\phi_{2}}{360}\right) \text {. }
$$

Equations (3) and (4) can be solved by finding Eq. (4) - Eq. (3) $\times\left(\lambda_{2} / \lambda_{1}\right)$ and rearranged to give

$$
D=\left(\frac{\lambda_{1} \lambda_{2}}{\lambda_{1}-\lambda_{2}}\right)\left(\left(n_{2}-n_{1}\right)+\frac{\left(\phi_{2}-\phi_{1}\right)}{360}\right) .
$$

Using $v=f \times \lambda$, we obtain

$$
D=\frac{v}{\Delta f}\left(\Delta n+\frac{\Delta \phi}{360}\right)
$$

TABLE I. Parameters used in example 1.

\begin{tabular}{lccccccc}
\hline \hline $\begin{array}{l}R \\
(\mathrm{~mm})\end{array}$ & $\begin{array}{c}v \\
(\mathrm{~mm} / \mu \mathrm{s})\end{array}$ & $\begin{array}{c}f_{1} \\
(\mathrm{kHz})\end{array}$ & $\begin{array}{c}\lambda_{2} \\
(\mathrm{~mm})\end{array}$ & $n_{1}$ & $\begin{array}{c}f_{2} \\
(\mathrm{kHz})\end{array}$ & $\begin{array}{c}\lambda_{2} \\
(\mathrm{~mm})\end{array}$ & $n_{2}$ \\
\hline 1500 & 1.50 & 200 & 7.50 & 200 & 201 & 7.462 & 201 \\
\hline \hline
\end{tabular}

where $\Delta f=f_{2}-f_{1}, \Delta n=n_{2}-n_{1}$, and $\Delta \phi=\phi_{2}-\phi_{1}$.

Knowing $D=v \times t$, we deduce the time delay $t$ as

$$
t=\frac{1}{\Delta f}\left(\Delta n+\frac{\Delta \phi}{360}\right) \text {. }
$$

If we impose the condition that $\Delta n \leqslant 1$, then Eq. (7) can be solved. This restriction on $\Delta n$ is imposed as follows.

- A distance $D$ is selected within which we require an unambiguous range measurement.

- Select a frequency $f_{1}$ within the bandwidth of the system, and its corresponding wavelength $\lambda_{1}$ [from Eq. (1)].

- Similarly, using Eq. (1), select frequency $f_{2}$ with its corresponding wavelength $\lambda_{2}$ such that the number of cycles is $n_{2}=n_{1}+1$.

Considering Eq. (6), the maximum range is achieved by this approach when $\Delta n=1$;

$$
R=\frac{v}{\Delta f} .
$$

Therefore, $R$ is the maximum unambiguous range that can be achieved using the two frequencies $f_{1}$ and $f_{2}$ as described above. As the phase differences $\Delta \phi$ will be unique within this range $R$, any distance within this range can be determined unambiguously.

\section{B. Example}

We demonstrate in this example how we could measure a range by using two frequencies and their related phase differences.

Consider an unambiguous range $R$; two frequencies $f_{1}$ and $f_{2}$ comprising integer number of cycles $n_{1}, n_{2}$ of wavelength $\lambda_{1}, \lambda_{2}$, respectively, as shown in Table I. Within this range $R$, consider a distance to target $d=1000.1234 \mathrm{~mm}$ we wish to estimate. Assume that a short $\mathrm{cw}$ pulse comprising these two frequencies $f_{1}$ and $f_{2}$ is sent toward the target at the distance $d$.

Using $f_{1}, f_{2}$ and Eqs. (1) and (6) for this distance $d$ gives an integer number $n_{1}=133$ and a residual fraction of cycle of $r_{1}=0.349786$ corresponding to a residual phase of $\phi_{1}$ $=125.923^{\circ}$. Similarly for the frequency $f_{2}$, we find the residual phase $\phi_{2}=5.953^{\circ}$ corresponding to $n_{2}=134$ and $r_{2}$ $=0.0165356$. Thus, $\Delta \phi=\phi_{2}-\phi_{1}=-119.970^{\circ}$.

We use this value in the formula given in Eq. (6), since $\Delta \phi$ is negative; this means $\Delta n=1$. Using $v$ from Table I, $\Delta f=1 \mathrm{kHz}$, and substituting into Eq. (7) give a first estimate of the range $\hat{d}_{f_{1} f_{2}}=1000.1233 \mathrm{~mm}$ (caret is used here to mean an estimate). The unambiguous range $R$ [Eq. (8)] is independent of the frequencies used depending only on the difference in frequency $\Delta f$. 
TABLE II. Parameters used in example 2

\begin{tabular}{lccccccc}
\hline \hline $\begin{array}{l}R \\
(\mathrm{~mm})\end{array}$ & $\begin{array}{c}v \\
(\mathrm{~mm} / \mu \mathrm{s})\end{array}$ & $\begin{array}{c}f_{3} \\
(\mathrm{kHz})\end{array}$ & $\begin{array}{c}\lambda_{3} \\
(\mathrm{~mm})\end{array}$ & $n_{3}$ & $\begin{array}{c}f_{4} \\
(\mathrm{kHz})\end{array}$ & $\begin{array}{c}\lambda_{4} \\
(\mathrm{~mm})\end{array}$ & $n_{4}$ \\
\hline 1500 & 1.5 & 210 & 7.142 & 210 & 300 & 5.0 & 300 \\
\hline \hline
\end{tabular}

Note that in practice such resolution may not be achievable and limitations must be considered. For example, if the accuracy of estimating the phase is within $\pm 0.5^{\circ}$, then the phases in the example above become $\phi_{1}=126.0$ and $\phi_{2}$ $=6.0$, giving $d=1000.0 \mathrm{~mm}$ implying an error of $0.1234 \mathrm{~mm}$. To get a finer resolution, we have to add other frequency components.

\section{Using multiple frequencies through a "Vernier approach"}

We demonstrate in this example how we could obtain a finer resolution in a range by using more than two frequencies and their related phase differences.

In Eq. (6), we imposed the condition that $\Delta n \leqslant 1$. The values of frequencies $f_{1}$ and $f_{2}$ were chosen to ensure this condition and to obtain a first estimate of the distance $\hat{d}_{f_{1} f_{2}}$, and an estimate of the time delay $\hat{t}_{f_{1} f_{2}}$ [Eq. (7)].

Introducing a third frequency $f_{3}$ as shown in Table II, such that $f_{3}-f_{1}=10 \times\left(f_{2}-f_{1}\right) ; f_{2}$ differs from $f_{1}$ by $1 \mathrm{kHz}$ and $f_{3}$ differs from $f_{1}$ by $10 \mathrm{kHz}$.

Again from Eq. (1), for $f_{3}$ and $d=1000.1234 \mathrm{~mm}, \phi_{3}$ $=6.219^{\circ}$, which we would measure as $6.5^{\circ}$. Thus, $\Delta \phi_{13}$ $=\phi_{3}-\phi_{1}=-119.5^{\circ}$. We add $360^{\circ}$ to give $240.5^{\circ}$. However, $\Delta n_{13}$ between frequencies $f_{1}$ and $f_{3}$ is now 7 (in fact, 6 since we have already added in $360^{\circ}$ to make the phase difference positive).

Using Eq. (6) with $\Delta \phi_{13}$ and different values of $\Delta n_{13}$ $(0-6)$ to get different distance estimate $\hat{d}_{f_{1} f_{3}}^{k}$, where $k=$ $0, \ldots, 6$, in this case.

Applying Eq. (6) recursively for $\Delta n_{13}=0, \ldots, 6$ to calculate $\left.\hat{d}_{f_{1} f_{3}}^{k}\right|_{k=0,6}$ selecting $\hat{d}_{f_{1} f_{3}}^{k}$ closest in value to $\hat{d}_{f_{1} f_{2}}$ as the optimum value $\hat{d}_{f_{1} f_{3}}=\left.1000.2083 \mathrm{~mm}\right|_{k=6}$. Hence, a new best time delay estimate $\hat{t}_{\left.f_{1} f_{3}\right|_{k=6}}$.

Note that the new best estimate distance is with an error of $0.0849 \mathrm{~mm}$.

If a fourth frequency $f_{4}$ is introduced, as shown in Table II, such that the $\Delta f_{14}=100.0 \mathrm{kHz}$, using Eq. (1) again gives $\phi_{4}=8.8848^{\circ}$ which we measure as $9^{\circ}$. Thus, $\Delta \phi_{14}=\phi_{4}-\phi_{1}$ $=-117^{\circ}$ which gives $\Delta \phi_{14}=249.5$ after adding $360^{\circ}$. Note that $\Delta n_{14}=66$ in this case.

Similarly, select the estimate $\hat{d}_{f_{1} f_{4}}(\Delta n=0,1,2, \ldots, 66)$ closest in value to $\hat{d}_{f_{1} f_{3}}$. This occurs at $\Delta n_{14}=66$ giving $\hat{d}_{f_{1} f_{4}}=1000.125 \mathrm{~mm}$. Taking this as the best estimate, the final error is $0.0016 \mathrm{~mm}=1.6 \mu \mathrm{m}$.

Note that the frequencies need not be in decadal scale variation; however, they should be selected within the bandwidth of the system. All the selected frequencies should be a multiple of the lowest difference ensuring an integer number of cycles; in this way, we ensure that the phase calculated by the discrete Fourier transform (DFT) will be correct (i.e., corresponding exactly to the frequency bins). Thus, this example is reminiscent of the operation of a Vernier gauge as follows:

- $\Delta \phi_{12}$, related to the frequencies $f_{1}$ and $f_{2}$, gives the first estimate of the distance $\hat{d}_{f_{1} f_{2}}$, hence $\hat{t}_{f_{1} f_{2}}$.

- A higher frequency $f_{3}$ is then used (decade difference) to measure the same range but with a finer resolution. So a more accurate approximation to the measured range is obtained $\hat{d}_{f_{1} f_{3}}$.

- Similarly, the measured range $\hat{d}_{f_{1} f_{4}}$ corresponding to $\Delta \phi_{14}$ within $f_{1}$ and $f_{4}$, will give the ultimate estimate of the measured range $d$.

- Consequently, the maximum distance and the minimum resolution achieved are determined by the choice of the frequencies $f_{1}, f_{2}, f_{3}$, and $f_{4}$.

An example algorithm calculating the phase at each frequency and outlining the iterative phase difference measurements is given in the Appendix.

\section{Phase offset measurement calibration}

In the numerical example above, it is assumed that all phases are accurately transmitted and received, with no phase error on transmission or reception, and that all frequencies have zero phase offset with respect to each other. In practice this is almost certainly not the case and phase offsets between frequencies should be accounted for as discussed below.

Considering two frequencies $f_{1}=200.0 \mathrm{kHz}$ and $f_{2}$ $=201 \mathrm{kHz}$, and assuming the speed of sound in water $(v$ $=1.5 \mathrm{~mm} / \mu \mathrm{s}$ ), from Eq. (8), the unambiguous range $R$ $=1500 \mathrm{~mm}$ and $\Delta n$ is 0 or 1 . Considering the above

$$
t=\frac{D}{v}=\frac{n+\phi / 360}{f} .
$$

Consider two distances $d_{1}, d_{2}$ corresponding to two "times" $t_{1}$ and $t_{2}$ such that the number of cycles $n$ is the same for both frequencies over these distances, and assuming the phase measured includes a phase offset for that frequency. As an example, suppose the unknown phase offset for $f_{1}$ is $10^{\circ}$, for $f_{2}$ is $30^{\circ}$, and assume $d_{1}=100 \mathrm{~mm}$.

From Eq. (9), the term $\left(n_{1}+\phi_{1} / 360\right)$ would be calculated as 13.33 cycles, where $\phi_{1}=120^{\circ}$. The "measured" $\phi_{1}$ $=120+10=130^{\circ}\left(\phi_{1 \text { measured }}=\phi_{1 \text { distance }}+\phi_{1 \text { offset }}\right)$.

Similarly, for $f_{2}$ we obtain 13.40 cycles, where $\phi_{2}$ $=144^{\circ}$. The measured $\phi_{2}=144+30=174^{\circ}$; from Eq. (7), $t_{1}$ $=12.22 \mu \mathrm{s}$. The actual time should be $6.66 \mu \mathrm{s}$.

Assume a second distance $d_{2}=200 \mathrm{~mm}$. Using Eq. (9), for $f_{1}$ we obtain 26.66 cycles, which gives $\phi_{1}=240^{\circ}$. The measured $\phi_{1}=240+10=250^{\circ}$. Similarly, for $f_{2}$ we obtain 26.80 cycles, which gives $\phi_{2}=288^{\circ}$. The measured $\phi_{2}$ $=288+30=318^{\circ}$. Thus, using Eq. (7), $t_{2}=18.88 \mu \mathrm{s}$. The actual time should be $13.33 \mu \mathrm{s}$.

A linear relationship could be deduced between $t$ and $d$ as follows:

$$
t=m \times d+c,
$$


where the slope $m=1 / 1.5=0.66$ is the speed of sound measured as $1 \mathrm{~mm}$ per $0.66 \mu$ s or $1 / 0.66=1.5 \mathrm{~mm} / \mu \mathrm{s}$. The intercept $c=5.55 \mu \mathrm{s}$ is a measure of the relative phase between $f_{1}$ and $f_{2}$. Since $\Delta f_{12}=10 \mathrm{kHz}, 1$ cycle is $100 \mu$ s long; consequently, the offset of $5.55 \mu \mathrm{s} \equiv 360 \times(5.55 / 100)=20^{\circ}$, which is equal to the relative phase (30-10) between the two frequencies. If we had known the phase offset between the two frequencies $\left(20^{\circ}\right)$, then in the calculation of times we would have obtained for $t_{1}$ a new phase difference of (174 $-130-20)=24^{\circ}$ giving a time for $t_{1}=6.66 \mu \mathrm{s}$.

Similarly, for $t_{2}$ we obtain a new phase difference of $(318-250-20)=48^{\circ}$ giving a time for $t_{2}=13.33 \mu \mathrm{s}$. Both $t_{1}$ and $t_{2}$ are now correct. Note the following.

- If we assumed $d_{1}$ was $100 \mathrm{~mm}$ but it was actually, say, $120 \mathrm{~mm}$ and that $d_{2}$ was $200 \mathrm{~mm}$ but it was actually $220 \mathrm{~mm}$, then we obtain the phase offset as $15.2^{\circ}$. The slope of Eq. (10) above, however, is unaffected. For example, such uncertainty may arise if the distance traveled by the wave within the transducers is not taken into consideration.

- If the temperature changes, so $v$ changes; this changes the slope of Eq. (10) but not the time intercept or the phase offset. For example, if $v=1.6 \mathrm{~mm} / \mu \mathrm{s}$, then equation Eq. (10) becomes $t=(1 / 1.6) \times d+5.55=0.625 \times d+5.55$.

\section{APPLICATION}

\section{A. Experiment}

To demonstrate this approach, a series of measurements was performed in a water tank measuring $1530 \times 1380$ $\times 1000 \mathrm{~mm}^{3}$. Two broadband ultrasonic transducers (Alba Ltd., Glasgow, UK), having a wide bandwidth with a center frequency between 100 and $130 \mathrm{kHz}$, operate as both transmitters (Tx) and receivers ( $\mathrm{Rx}$ ) of ultrasound with a beam width of around $10^{\circ}$ at the center frequency, where $-3 \mathrm{~dB}$ bandwidth is $99 \mathrm{kHz}(72-171 \mathrm{kHz})$. These were mounted on a trolley, movable in the $X-Y$ directions. Linear encoders (Newall Ltd., Leicester, UK) were used to measure displacement of the rails in the $x$-direction and a software provided readouts of transducer positions. The temperature in the tank was measured by thermocouples calibrated using a quartz thermometers which are traceable to national standard. They were positioned on the four sides panels of the tank and recorded $19.80 \pm 0.05{ }^{\circ} \mathrm{C}$ during the experiment. The experiment setup is shown in Fig. 1. The transmitter was driven by a $20 \mathrm{~V}$ peak-to-peak waveform consisting of short $\mathrm{cw}$ pulse comprising four frequencies $(70,71,80$, and $170 \mathrm{kHz})$. A modular system comprising a 16-bit arbitrary waveform generator (Ztec ZT530PXI) and a 16-bit digital storage oscilloscope (Ztec ZT410PXI) was used for transmit/receive process. Software was used to control signal transmission and acquisition. Distances between $\mathrm{Tx}$ and $\mathrm{Rx}$ of 305.800, $345.778,481.128,535.131,535.252,624.515,759.182$, and $862.887 \mathrm{~mm}$ were selected to be within an unambiguous range of $R \approx 1500 \mathrm{~mm}$ [Eq. (8)], as set by the linear encoders. A set of ten signals, noise free signal and signals with different signal to Gaussian noise ratios (SNR $=-20,-10$, $-5,-2,0,2,5,10,20 \mathrm{~dB})$, was transmitted at each distance

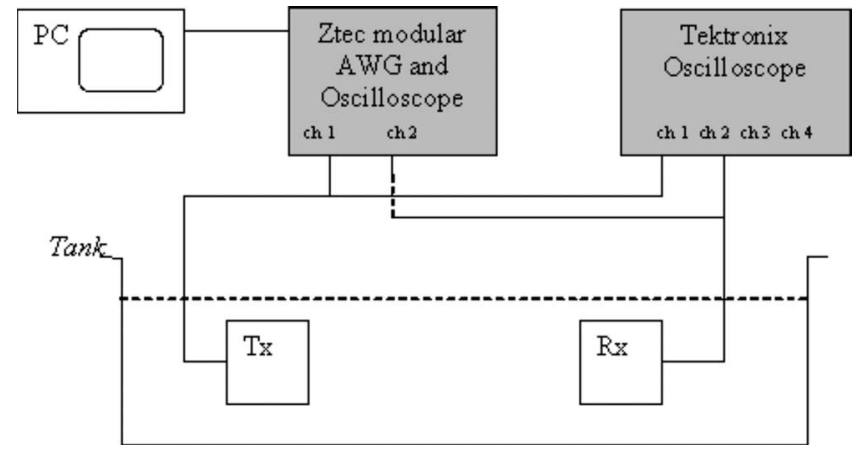

FIG. 1. Schematic diagram of the experimental setup.

described above. Note that the added Gaussian noise was generated by software and added to the original signal in each case. Before transmitting, each signal was multiplied by a Tukey window (cosine-tapered window with a 0.2 taper ratio) to reduce the "turn on" and "turn off" transients of the transducers. At each distance, three repeat pulses were transmitted and received for each SNR and each distance. Furthermore, 60 repeat pulses were transmitted and received while keeping the distance constant at $862.887 \mathrm{~mm}$ to assess the repeatability of the system (see Fig. 3). The sampling frequency $F_{s}$ was set to $10 \mathrm{MHz}$, giving a number of samples $N=20000$ and a 2 ms pulse length. A DFT was then applied to the received pulses to obtain the magnitude and phase information for each of the four frequency components, using a window of $[N / 2+1: N]$ for each received signal. This gave a resolution $F_{s} / N / 2=1 \mathrm{kHz}$ which was consistent with the smallest step between the four frequencies comprised in the pulse.

Figure 2(a) shows the noise free transmitted (top) and received (bottom) signals when Tx and Rx were $305.800 \mathrm{~mm}$ apart, while Fig. 2(b) shows transmitted (top) and received (bottom) signal in the greatest noise case SNR $(-20 \mathrm{~dB})$. Note the DFT reports phase with respect to cosine, whereas sine waves were used in this experiment. Sine waves are returned with a phase of $-90^{\circ}$ relative to cosine waves by the DFT. This was not an issue, since relative phase differences were used. Using the phase for each distance obtained by the DFT, the phase-based time delay algorithm given in the Appendix was applied to obtain the corresponding estimated times for each phase difference $\Delta \phi_{12}, \Delta \phi_{13}, \Delta \phi_{14}, \Delta \phi_{23}$, $\Delta \phi_{24}$, and $\Delta \phi_{34}$, for the pairs $f_{1} f_{2}, f_{1} f_{3}, f_{1} f_{4}, f_{2} f_{3}, f_{2} f_{4}$, and $f_{3} f_{4}$, respectively.

Using a simple calculation of the first estimate by $t_{12}$ $=\Delta \phi_{12} /\left(f_{2}-f_{1}\right)$ as a first estimate using Eq. (7) gave corresponding estimated times $\hat{t}_{12}, \hat{t}_{13}, \hat{t}_{14}, \hat{t}_{23}, \hat{t}_{24}$, and $\hat{t}_{34}$, respectively. For each distance, $\hat{t}_{14}$ should be the best estimate (i.e., the greatest $\Delta f$ ). The sensitivity of the algorithm to noise was reduced by the following.

- Limitation of the noise beyond the sensitivity of the transducer. The transducer act with its bandwidth as a bandpass filter.

- Signals were averaged 32 times during acquisition. When acquiring the signal with averaging, this reduces the noise level.

- The DFT operates on sine waves preferentially, even in the 

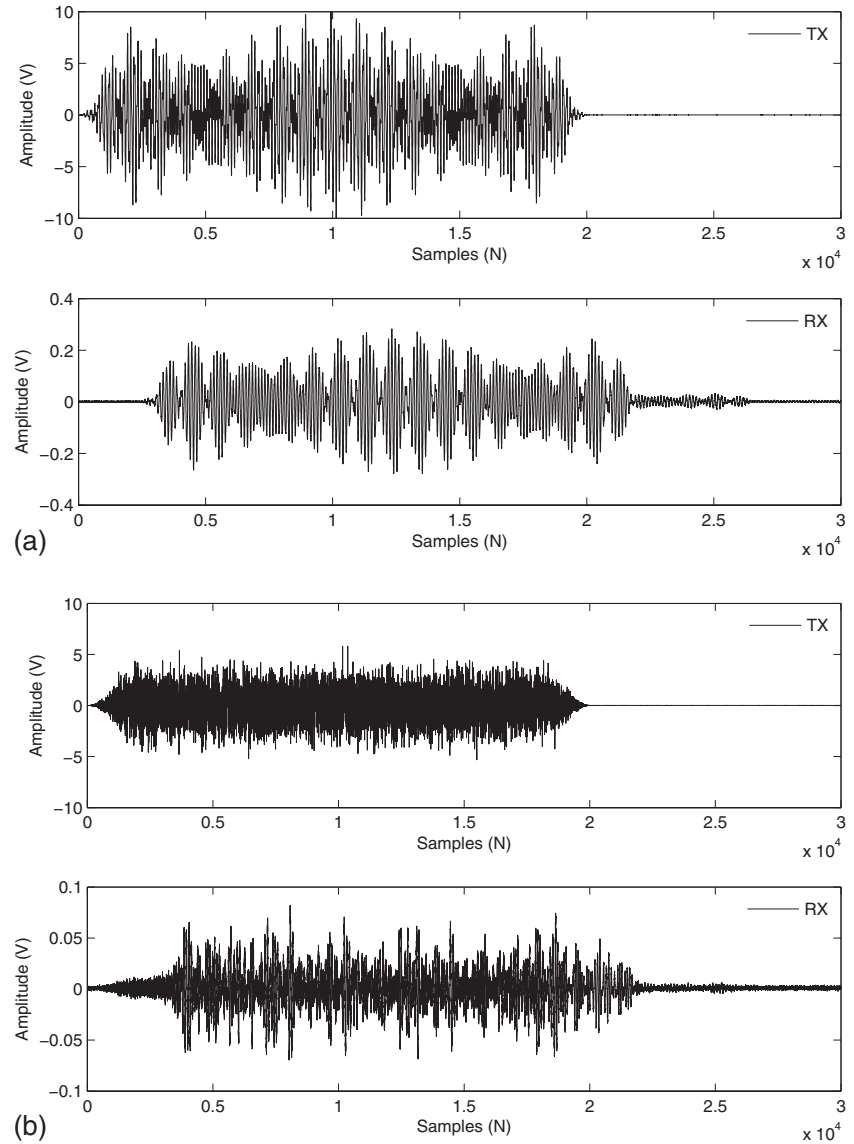

FIG. 2. Examples of transmitted and received short four components $\mathrm{cw}$ signals. Without (upper) and with (lower) added noise (-20 dB).

presence of noise. As the transmitted frequencies are known, when applying the DFT for the received signal, we will look just for those frequencies; hence any other frequencies are seen as noise and will not affect the frequencies of interest.

- Gradient estimation using a least squares fitting is noise tolerant. When doing the least squares estimation, any outlier which is basically noise will be removed.

\section{B. Results and discussion}

The best estimated time delays $\hat{t}_{14}$ for the eight different distances and using different signal-to-noise ratios are shown in Table III. For each distance, ten estimations were given corresponding to different signal-to-noise ratios. The column 2 corresponding to $\hat{t}_{14, \text { Org }}$, represents the estimated times when no noise was added to the signal. Using $\hat{t}_{14, \text { Org }}$ as best estimate time reference related to noise free signal, we can see that the technique became more robust to noise when the signal-to-noise ratio become greater than $2 \mathrm{~dB}$. The variation is within 50, 30, 20, and $10 \mu$ s when the SNRs are 2, 5, 10, and $20 \mathrm{~dB}$, respectively.

For the two rows in Table III corresponding to the distances 535.131 and $535.252 \mathrm{~mm}$, where the displacement is about $120 \mu \mathrm{m}$, the estimated time for this displacement is about $120 \pm 20 \mathrm{~ns}$ when the SNR is greater than $-5 \mathrm{~dB}$. As the sampling frequency used in this experiment was $10 \mathrm{MHz}$, using the conventional method (e.g., crosscorrelation) to estimate the time delay, we would not get a best time resolution of less than $1 / 10^{7}=100 \mathrm{~ns}$, which corresponds to a displacement of $150 \mu \mathrm{m}$, when assuming a speed of sound of $1500 \mathrm{~m} / \mathrm{s}$. Hence, such displacement could not be measured without interpolation or oversampling. Moreover, if the cross-correlation approach was used to estimate the time delay in this experiment, all the measurements in Table III would be an integer of the sampling rate; in this case, $100 \mathrm{~ns}$, as the technique is samplingfrequency dependent.

Figure 3(a) shows the time-distance plots, with the least squares fitting (linear regression), corresponding to the most noisy case $(\mathrm{SNR}=-20 \mathrm{~dB})$. The error plot (bottom) shows the residuals with a norm of about $170 \mu$ s. Figure 3(b) shows the case of the best SNR $(20 \mathrm{~dB})$ and the corresponding residual error norm equal to $0.268 \mu \mathrm{s}$. The estimated time shown in $y$-axis corresponds to the best estimate $\hat{t_{14}}$ which is based on the phase difference between the two components $f_{1}=70 \mathrm{kHz}$ and $f_{4}=170 \mathrm{kHz}$. The time delay estimation values with and without SNR ratios can be seen to be almost identical for each distance except for the two lowest SNR -20 and $-10 \mathrm{~dB}$. The data plotted in Fig. 3(b) (top) corresponds to a linear equations of the form $t=m d+c$, where $c$ is a measure of the phase offset for the two frequencies equal to $c \times\left(f_{4}-f_{1}\right) \times 360$ and $1 / m=v(\mathrm{~mm} / \mu \mathrm{s})$ is the estimated sound velocity in water, where $m$ is the slope. Solving $t$ $=m d+c$ by least squares fitting gave the best estimate $m$, where $(1 / m)$ is the speed of sound $v$ in water.

Table IV represents the results after a least squares fitting estimation, showing the estimated sound velocity and the related offset for each situation (original and $-20-20 \mathrm{~dB}$

TABLE III. The estimated time delays for the eight distances with different SNR in Rx.

\begin{tabular}{|c|c|c|c|c|c|c|c|c|c|c|}
\hline \multirow{2}{*}{$\begin{array}{c}\text { Distance } \\
(\mathrm{mm}) \\
d_{\text {meas }} \\
\end{array}$} & \multicolumn{10}{|c|}{ Estimated time $(\mu \mathrm{s})$} \\
\hline & $\hat{t}_{14, \text { Org }}$ & $\hat{t}_{14,-20 \mathrm{~dB}}$ & $\hat{t}_{14,-10 \mathrm{~dB}}$ & $\hat{t}_{14,-5 \mathrm{~dB}}$ & $\hat{t}_{14,-2 \mathrm{~dB}}$ & $\hat{t}_{14,0 \mathrm{~dB}}$ & $\hat{t}_{14,2 \mathrm{~dB}}$ & $\hat{t}_{14,5 \mathrm{~dB}}$ & $\hat{t}_{14,10 \mathrm{~dB}}$ & $\hat{t}_{14,20 \mathrm{~dB}}$ \\
\hline 305.800 & 242.05714 & 328.14350 & 241.40850 & 241.86129 & 241.95502 & 241.99356 & 242.01367 & 242.03214 & 242.04162 & 242.04872 \\
\hline 345.778 & 269.18036 & 366.64520 & 268.79819 & 269.06062 & 269.12812 & 269.14930 & 269.16954 & 269.17011 & 269.18868 & 269.18072 \\
\hline 481.128 & 360.43477 & 347.04536 & 360.06666 & 360.31378 & 360.37864 & 360.39371 & 360.41009 & 360.40717 & 360.42855 & 360.43632 \\
\hline 535.131 & 396.63307 & 492.90101 & 396.20888 & 396.50459 & 396.56616 & 396.59312 & 396.60089 & 396.62446 & 396.63076 & 396.62781 \\
\hline 535.252 & 396.76493 & 493.05600 & 396.31506 & 396.62782 & 396.69481 & 396.73015 & 396.72628 & 396.73571 & 396.75997 & 396.75431 \\
\hline 624.515 & 456.86671 & 442.81417 & 456.07791 & 456.61695 & 456.74634 & 456.78030 & 456.81829 & 456.82773 & 456.84870 & 456.85793 \\
\hline 759.182 & 547.80206 & 522.71162 & 537.17804 & 547.60860 & 547.70913 & 547.73865 & 547.75749 & 547.78524 & 547.79431 & 547.80029 \\
\hline 862.887 & 617.79228 & 391.21736 & 607.22052 & 617.63769 & 617.70199 & 617.72574 & 617.75122 & 617.78719 & 617.78503 & 617.80452 \\
\hline
\end{tabular}



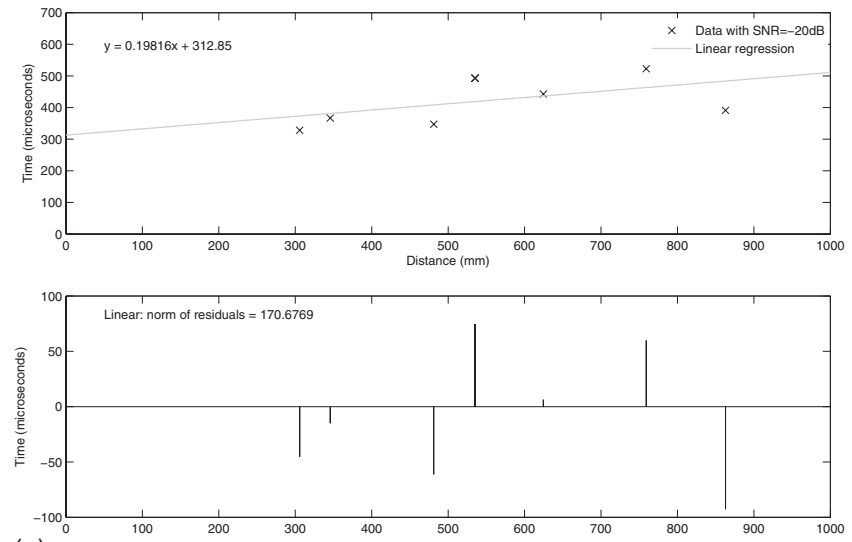

(a)
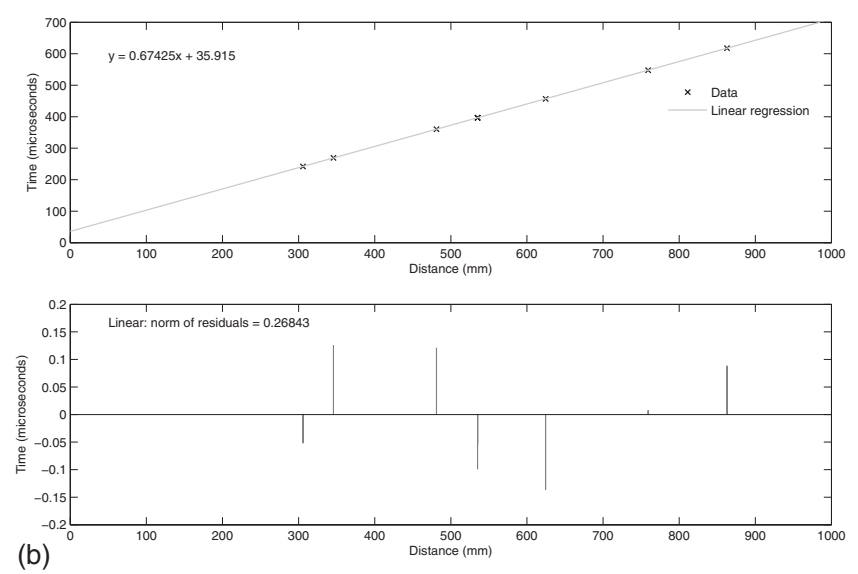

FIG. 3. Estimated times versus distances, $-20 \mathrm{~dB}$ SNR case (top), noise free case (bottom), and their corresponding least squares fitting showing the residual errors.

SNR). The speed of sound is estimated to be $1483 \mathrm{~m} / \mathrm{s}$ $\pm 0.2 \mathrm{~m} / \mathrm{s}$ when the $\mathrm{SNR}$ is greater or equal to $-5 \mathrm{~dB}$ and an average of temperature in the tank of $19.87^{\circ} \mathrm{C}$.

The speed of sound in pure water with similar temperature was reported in Ref. 16 to be $1481.727 \mathrm{~m} / \mathrm{s}$ and in Ref. 17 to be $1482.36 \mathrm{~m} / \mathrm{s}$. Figure 4 shows the speed of sound variation versus different SNR ratios in a logarithmic scale; the approach can be seen to be less sensitive to noise when the SNR is greater than $-10 \mathrm{~dB}$.

Table $\mathrm{V}$ shows the measured distances and the corresponding estimated distances using the equations of Table

TABLE IV. Least squares fitting equations for different SNR in Rx and the corresponding estimated speed of sound from the slope.

\begin{tabular}{cccc}
\hline \hline $\begin{array}{c}\text { Curve } \\
(\mathrm{dB})\end{array}$ & $t=m d+C$ & $\begin{array}{c}\hat{v} \\
(\mathrm{~mm} / \mu \mathrm{s})\end{array}$ & Phase offset \\
\hline Noise free & $t=0.674182 \times d+35.901766$ & 1.483279 & 212.463589 \\
-20 & $t=0.198160 \times d+312.848259$ & 5.046422 & 102.537332 \\
-10 & $t=0.653855 \times d+44.229354$ & 1.529392 & 152.256749 \\
-5 & $t=0.674171 \times d+35.798682$ & 1.483303 & 208.752539 \\
-2 & $t=0.674186 \times d+35.871635$ & 1.483270 & 211.378847 \\
0 & $t=0.674182 \times d+35.901766$ & 1.483279 & 212.463589 \\
2 & $t=0.674197 \times d+35.911676$ & 1.483247 & 212.820339 \\
5 & $t=0.674244 \times d+35.900430$ & 1.483142 & 212.415469 \\
10 & $t=0.674221 \times d+35.926900$ & 1.483193 & 213.368412 \\
20 & $t=0.674250 \times d+35.914968$ & 1.483130 & 212.938860 \\
\hline \hline
\end{tabular}

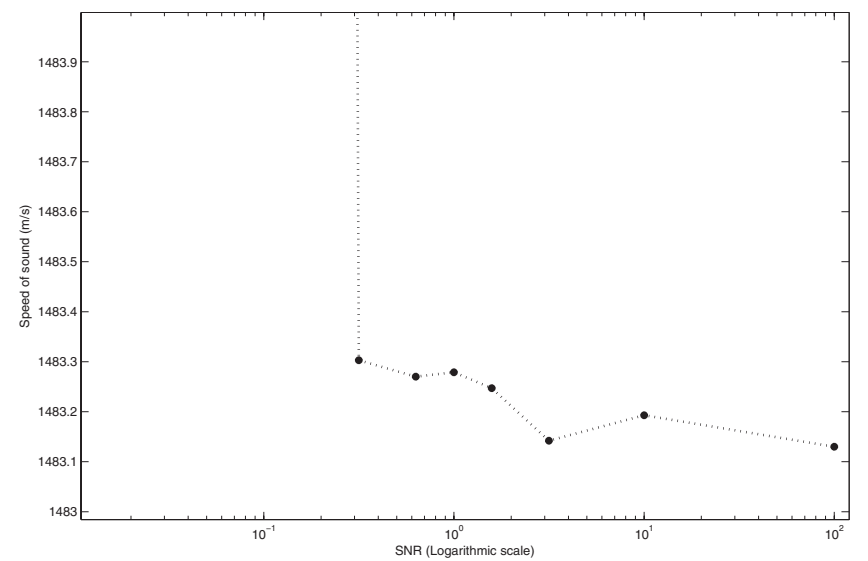

FIG. 4. Estimated speed of sound versus different SNRs $(\mathrm{SNR}=$ -5 to $20 \mathrm{~dB})$.

IV. In this case, the estimated distance is given by $\hat{d}=(t$ $-c) \times v$, where $t$ is the estimated time corresponding to the best estimate $\hat{t_{14}}, c$ is the time offset due to the time delay through the transducers, and $v$ is the estimated sound velocity in water. The differences between the measured and estimated distances lie within the range 5-315 $\mu \mathrm{m}$ (see Table $\mathrm{V})$ when the SNR is greater than $-10 \mathrm{~dB}$. The maximum difference represents $1 / 50$ of the wavelength at the central frequency of the transducer $(100 \mathrm{kHz})$.

\section{CONCLUSION}

In this paper, a time delay estimation approach based on phase differences between components of the received short $\mathrm{cw}$ pulse signal is demonstrated. A validation underwater experiment showed that a resolution greater than $1 / 50$ of the wavelength was achievable. Using local phase difference information, no ambiguity in phase measurement arises; hence there is no need to use the Chinese remainder theorem or a coherent local oscillator to overcome the wellknown phase ambiguity problem. As phase information is usually regarded to be useless in most correlator-based techniques, the approach developed in this paper is distinctive. Compared to correlator-based approaches, which have an estimation accuracy on the order of the used bandwidth, the suggested approach is not limited by the bandwidth but only by the ability to measure phase differences accurately. This leads to much improved performance once the SNR is sufficiently high. This approach is tolerant to additive Gaussian noise when the SNR is acceptable. Consequently, the technique offers the potential to outperform animals in subwavelength measurements. Although a bat can achieve a resolution of $20 \mu \mathrm{m}$ in air, ${ }^{15}$ potentially we would get a resolution of $4 \mathrm{~mm} / 360$ $=11 \mu \mathrm{m}$. As a new approach, we expect that the algorithm will be improved to the point where one degree of phase can be resolved, suggesting that performance similar to the bat will be achieved by such technology in the near future.

\section{ACKNOWLEDGMENTS}

This work was undertaken as part of the Biologically Inspired Acoustic Systems (BIAS) project, funded by the RCUK Basic Technology Programme Grant Number EP/ 
TABLE V. The measured (using the encoders) and the estimated distances with different SNR in Rx.

\begin{tabular}{|c|c|c|c|c|c|c|c|c|c|c|}
\hline \multirow{2}{*}{$\begin{array}{l}\text { Measured } \\
\quad(\mathrm{mm}) \\
d_{\text {meas }}\end{array}$} & \multicolumn{10}{|c|}{$\begin{array}{l}\text { Estimated distances } \\
\qquad(\mathrm{mm})\end{array}$} \\
\hline & $\hat{d}_{\text {org }}$ & $\hat{d}_{-20 \mathrm{~dB}}$ & $\hat{d}_{-10 \mathrm{~dB}}$ & $\hat{d}_{-5 \mathrm{~dB}}$ & $\hat{d}_{-2 \mathrm{~dB}}$ & $\hat{d}_{0 \mathrm{~dB}}$ & $\hat{d}_{2 \mathrm{~dB}}$ & $\hat{d}_{5 \mathrm{~dB}}$ & $\hat{d}_{10 \mathrm{~dB}}$ & $\hat{d}_{20 \mathrm{~dB}}$ \\
\hline 305.800 & 305.785 & 77.186 & 301.564 & 305.653 & 305.677 & 305.691 & 305.700 & 305.722 & 305.707 & 305.723 \\
\hline 345.778 & 346.017 & 271.482 & 343.453 & 345.998 & 345.982 & 345.971 & 345.979 & 345.972 & 345.972 & 345.963 \\
\hline 481.128 & 481.372 & 172.573 & 483.039 & 481.354 & 481.331 & 481.312 & 481.311 & 481.289 & 481.298 & 481.307 \\
\hline 535.131 & 535.065 & 908.622 & 538.314 & 535.036 & 535.007 & 535.005 & 534.991 & 535.004 & 534.993 & 534.984 \\
\hline 535.252 & 535.260 & 909.404 & 538.477 & 535.218 & 535.198 & 535.209 & 535.177 & 535.169 & 535.185 & 535.171 \\
\hline 624.515 & 624.408 & 655.862 & 629.877 & 624.200 & 624.270 & 624.280 & 624.308 & 624.294 & 624.308 & 624.313 \\
\hline 759.182 & 759.290 & 1059.059 & 753.911 & 759.169 & 759.193 & 759.196 & 759.193 & 759.197 & 759.198 & 759.192 \\
\hline 862.887 & 863.105 & 395.483 & 861.034 & 863.043 & 863.011 & 863.007 & 863.011 & 863.020 & 863.007 & 863.017 \\
\hline
\end{tabular}

C523776/1. The BIAS project involves collaboration between the British Geological Survey, Leicester University, Southampton University, Leeds University, Edinburgh University, and Strathclyde University. This paper is published with the permission of the Executive Director of the British Geological Survey (NERC).

\section{APPENDIX: TIME DELAY ESTIMATION ALGORITHM USING PHASE DIFFERENCES}

Input: Set of phases $\left(\phi_{1}, \phi_{2}, \phi_{3}, \phi_{4}\right)$ and frequencies $\left(f_{1}, f_{2}, f_{3}, f_{4}\right)$

Output: Set of estimated time delay $\left(\hat{t}_{12}, \hat{t}_{13}, \hat{t}_{14}, \hat{t}_{23}, \hat{t}_{24}, \hat{t}_{34}\right)$

foreach Received signal, $k$ do

Calculate DFT $\left(f_{1}, f_{2}, f_{3}, f_{4}, k\right)$;

Get $\phi_{1}, \phi_{2}, \phi_{3}, \phi_{4}$

end

foreach $(i, j)=(1,3) ;(1,4) ;(2,3) ;(2,4) ;(3,4)$ do

$\Delta \phi_{i j}=\phi_{j}-\phi_{i}$;

if $\left(\left(\Delta \phi_{i j}\right)<0\right)$ then

end

$$
\Delta \phi_{i j}=\Delta \phi_{i j}+360.0 \text {; }
$$

end

$\Delta \phi_{12}=\frac{\Delta \phi_{12}}{360.0}, \Delta f_{12}=f_{2}-f_{1}, \hat{t}_{12}=\frac{\Delta \phi_{12}}{\Delta f_{12}}, \hat{t}_{\text {old }}=\hat{t}_{12}$

foreach $(i, j)=(1,3) ;(1,4) ;(2,3) ;(2,4) ;(3,4)$ do

if $\left(\left(\hat{t}_{\text {old }} \times \Delta f_{i j}\right)<1.0\right)$ then

$\hat{t}_{\text {new }}=\frac{\Delta \phi_{i j}}{\Delta f_{i j}} ;$

$\operatorname{nmin}=-1$;

end

$\min 1.0 e+06$;

for each $n=0$ to 1000 do

Calculate $\hat{t}_{i j}=\left(n+\Delta \phi_{i j}\right) / \Delta f_{i j}$;

$\Delta t=\left|\hat{t}_{i j}-\hat{t}_{\text {old }}\right|$;

if $(\Delta t<\min )$ then

$\min =\Delta t$;

$\hat{t}_{\text {new }}=\hat{t}_{i j}$;

$\operatorname{nmin}=n$;

end

end

end
${ }^{1}$ G. H. Knapp and G. C. Carter, "The generalised correlation method for estimation of time delay," IEEE Trans. Acoust., Speech, Signal Process. 24, 320-327 (1976).

${ }^{2}$ G. C. Carter, "Time delay estimation for passive sonar signal processing," IEEE Trans. Acoust., Speech, Signal Process. 29, 463-470 (1981).

${ }^{3}$ G. C. Carter, "Coherence and time delay estimation," in Signal Processing Handbook, edited by C. H. Chen (Dekker, New York, 1998), pp. 443-482.

${ }^{4}$ R. E. Boucher and J. C. Hassab, "Analysis of discrete implementation of generalised cross-correlator," IEEE Trans. Acoust., Speech, Signal Process. ASSP-29, 609-611 (1981).

${ }^{5}$ J. Chen, J. Benesty, and Y. A. Huang, "Time delay estimation via linear interpolation and cross correlation," IEEE Trans. Speech Audio Process. 12, 509-519 (2004).

${ }^{6}$ J. Chen, J. Benesty, and Y. A. Huang, "Time delay estimation in room acoustic environments: An overview," EURASIP J. Appl. Signal Process. 2006, 1-19.

${ }^{7}$ L. Belostotski, T. L. Landecker, and D. Routledge, "Distance measurement with phase stable $\mathrm{CW}$ radio link using the Chinese remainder theorem," Electron. Lett. 37, 521-522 (2001).

${ }^{8}$ C. E. Towers, P. D. Towers, and D. C. Jones Julian, "The efficient Chinese remainder theorem algorithm for full-field fringe phase analysis in multiwavelength interferometry," Opt. Express 12, 1136-1143 (2004).

${ }^{9} \mathrm{X}$. Xiang-Gen and L. Kejing, "A generalized Chinese remainder theorem for residue sets with errors and its application in frequency determination from multiple sensors with low sampling rates," IEEE Signal Process. Lett. 12, 768-771 (2005).

${ }^{10}$ O. Goldreich, D. Ron, and M. Sudan, "Chinese remaindering with errors," IEEE Trans. Inf. Theory 93, 1330-1338 (2000).

${ }^{11}$ A. K. T. Lee, J. Lucas, and L. E. Virr, "Microcomputer-controlled acoustic rangefinding technique," J. Phys. E: J. Sci. Instrum. 22, 52-58 (1989).

${ }^{12}$ M. Yang, S. L. Hill, B. Bury, and J. O. Gray, "A multifrequency AMbased ultrasonics system for accuracy distance measurement," IEEE Trans. Instrum. Meas. 43, 861-866 (1994).

${ }^{13} \mathrm{H}$. Hartridge, "Acoustical control in the flight of bats," Nature (London) 156, 490-494 (1945); 156, 692-693 (1945)

${ }^{14}$ J. A. Simmons, "The resolution of target range by echolocating bats," J. Acoust. Soc. Am. 54, 157-173 (1973).

${ }^{15}$ W. L. Whitlow and J. A. Simmons, "Echolocation in dolphins and bats," Phys. Today 60(9), 40-45 (2007).

${ }^{16}$ V. A. Del Grosso and C. W. Mader, "Speed of sound in pure water," J. Acoust. Soc. Am. 52, 1442-1446 (1972).

${ }^{17} \mathrm{~N}$. Bilaniuk and G. S. K. Wong, "Speed of sound in pure water as a function of temperature," J. Acoust. Soc. Am. 93, 1609-1612 (1993). 